DOI: 10.12731/2227-930X-2019-1-64-81

UDC 004.8

\title{
GOVERNANCE OF ARTIFICIAL INTELLIGENCE IN KSA (NEOM AS A MODEL)
}

Rana Ashehri

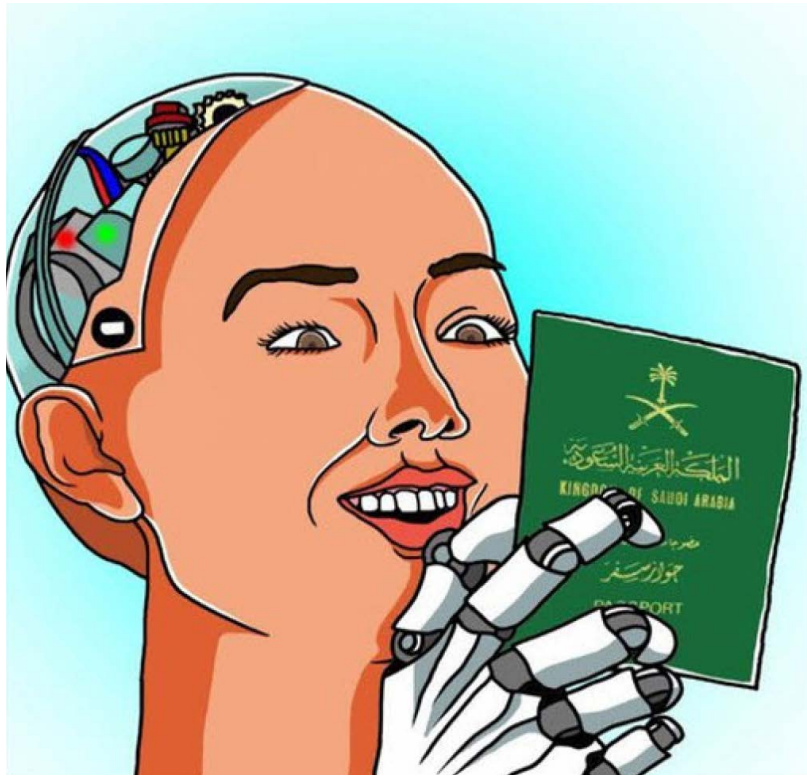

This paper deals with the issue of the governance of artificial intelligence from two aspects; the first one is the artificial intelligence as an essential component of the life of today's world, whether at the level of individuals or societies; its history, effects, benefits, fields and fears arising from it, and consequently the importance of the principal of the governance of artificial intelligence in maximizing advantages and preventing disadvantages. The second aspect deals with the efforts of the Kingdom of Saudi Arabia in the field of the governance of artificial intelligence as one of the leading countries in Middle East in implementing and investing of artificial intelligence applications for the 
sake of Saudi society and individuals. As well as to provide opportunities for companies and institutions from entire world to collaborate inside the kingdom and benefit the world. The Kingdom of Saudi Arabia's efforts are expressed through talking about the Saudi's giant project NEOM, through the discussion of the founding council's vision and message, as well as NEOM's features and sectors.

Keywords: Artificial Intelligence; Governance; NEOM; Technology.

\section{Introduction}

If steam was the basis of the industrial revolution of the eighteenth and nineteenth centuries, Artificial intelligence (AI) is being considered as the nucleus of the technological revolution that began in the mid- $20^{\text {th }}$ century.

It is no longer enough for man now to dispense with working with his bare hands and primitive animals he owns by machines; man, of this age requires machine to go much further.

In spit of his wide steps in artificial intelligence, man has stumbled on his way to the ideal full-thinking machine with shocking facts that have made many interested observers in front of this expected accomplishment full of fears and dread feelings related to moral considerations and the concept of divine synthesis between intelligence and virtue.

Besides worries about what these intelligent machines can do, there are new concerns about the impact of these machines on the labor, in the light of the fact that one intelligent machine is capable of replacing dozens of workers at lower costs, which raises questions about the human labor future and the level of unemployment expected.

However, all of the above does not simply mean that we have to abandon the AI development activities and to put it aside! for time is not backward, and evolution is inevitable. It means to control the course of AI project activates wisely and collectively, and not leaving it to individuals' judgments by creating a set of rules, criteria and regulations under which the AI development program is managed and controlled in order to achieve the utmost level of performance while avoiding all - or most of - risks and disadvantages expected, i.e., artificial intelligence governance. 
One of the most important countries concerned with this project is the Kingdom of Saudi Arabia, which is doing its best to keep pace with the global development and the efforts exerted in this field worldwide, where NEOM project is one of the most important manifestations of the kingdom's efforts in this field.

This paper is discussing two main points:

- The importance and necessity of Artificial Intelligence Governance as a concept, - the Kingdom of Saudi Arabia as a model.

- The efforts exerted by the Kingdom of Saudi Arabia in adopting and harnessing AI-based systems in the life of Saudi people in general and NEOM project as a model.

\section{History of Artificial Intelligence}

The field of artificial intelligence research was founded as an academic discipline in the middle of fifties, after great efforts made by several scientists from various fields in fifties and forties at the height of world obsession of modern science. Scientists were trying to find out the answer of the question "Is it possible to create an artificial brain?". (AlanTuring.net)

So, the Turing Test was the first serious proposal in the philosophy of artificial intelligence, which was devised by Alan Turing ("Smith", (n.d.)).

The basic notion Turing test depended on was: if the machine (computer) could carry a conversation that the observer can not distinguish from a conversation with a human, then it was reasonable to say that the machine was thinking. (Norvig \& Russell (2003)).

However, the real birth of AI was in the Dartmouth Conference in 1956, the moment that AI gained its name, mission and first success (Crevier (1993)).

In Dartmouth Conference; the "Logic Theorist" was debuted by Newell and Simon, and the Artificial Intelligence was proposed for the first time by John McCarthy, the scientist who convinced the conference's participants to accept the name "Artificial Intelligence" as the name of this field (McCorduck 2004, p. 114).

The years between 1956-1974 were mentioned later as the golden years of AI, for they were a real ear of discovery. In those years, a big 
number of programs were developed to enable computers to solve algebra word problems, proving theorems in geometry and learning English. Audience in that time considered this as marvelous and amazing for they didn't believe till that time that such a thing is possible (Crevier 1993, p. 52-107).

This ambience prompted researchers to predict that a fully intelligent machine would be built in less that 20 years later. Where it was the first time that governmental agencies like DARPA (The Defense Advanced Research Projects Agency of the United States) poured money in AI field. (Crevier 1993, p. 52-107).

\section{In simple words, what does AI mean?}

Artificial Intelligence is the activity to making machines intelligent, to work with foresight in the given environment. (Nilsson, 1980). The core stone of AI - for humans - is to make computers capable for understanding humans' thoughts and decisions through a set of advanced programs, i.e., through synthesizing software and hard work for better results with foresight. (Nilsson, 1980).

\section{Domains of Artificial Intelligence}

Many aspects in humanity life have been strongly affected or at least touched by AI, it may be useful to point to some of them for example, but not limited to.

\subsection{Education}

Human teachers are the core of the educational process; however, they need AI applications as an instrument to develop their educational activities and make them more effective, either for K-12 or university education. (Stone, P.; 2016)

The start of using robots for educational purposes was Lego Mindstorms Kits developed by the MIT Media Lab in eighties. Other examples were invented later such as Ozobot and Cubelets. (Stone, P.; 2016)

\subsection{Transportation}

One of the most important domains of AI-based applications is transportation. The notion of self-driving vehicles using radars, sen- 
sors, cameras and videos is amazing to most people now. Furthermore, the autopilot existed now in planes takes the job of performing what the pilot should do by himself for long hours during flights accurately, efficiently and effortlessly, where it became a very vital device in preventing accidents in most cases for it is capable of measuring height, speed and other important diameters, joining them together and make the right decision.

GPS has been most effective and reliable for all users helping in the specification of locations of transport destinations. (Liao, Patterson, Fox \& Kautz, 2007). Where sensors sympathized with intelligent applications to be able to identify people in automated vehicles, can save lives by reducing crashes and managing traffic congestions (Wagner, Baker, Goodin, Maddox, 2014).

\subsection{Games}

Chess, Checkers and Poker are some examples of games resulted from AI. When IBM "Deep Blue" won world chess champion Garry Kasparov, the world knew to what extent these machines could go in intelligence.

Games in general were an important aspect that benefited from AI, for it makes them more appealing (Nilsson, 2010).

\subsection{Health Care}

The importance of AI in the domain of health, was mentioned in the monthly magazine "Medical Device \& Diagnostic Industry" one of the most important medical magazines all over the world (Nilsson; 2010).

Artificial intelligence technology is a vital part of medical systems and support. However, poor human-computer interaction methods and the inherent difficulties and risks of implementing technologies in such a complex system have slowed realization of AI participation in healthcare (Leigh Anne Olsen; 2007).

\subsection{Public Safety and Security}

It is true the AI-based systems haven't provided what is expected from them in security sector yet. However, we have a group of distinguished AI applications that play an important role in security purposes, like: 


\subsubsection{Cyber Attacks and software Errors/Failures}

AI-based systems have been efficiently resisting attacks performed by humans to working systems, as well as errors that the software powering computers is subjected to. New labs, Cyber Security Lab, for instance, is concerned not just with human hackers, but with the ways in which AI itself may turn against systems.

\subsubsection{Security and Crime Prevention}

In 1995, New York Police Department's Computer Statistics used an early tool of AI depended on underlying software tools as the first of predictive Policing.

Predictive analytics and other AI-powered crime analysis tools have made significant strides since then. Avata Intelligence for example, has been using AI with game theory to predict when terrorists or other threats will strike a target (Artificial Intelligence and Security; Tech Emergence. Com).

Some other AI-based applications, such as robots used to deal with highly hostile situations like explosive spots or dangerous epidemics, are providing an extremely valuable services for humanity in the field of security.

The following graph illustrates domains of AI with more details.

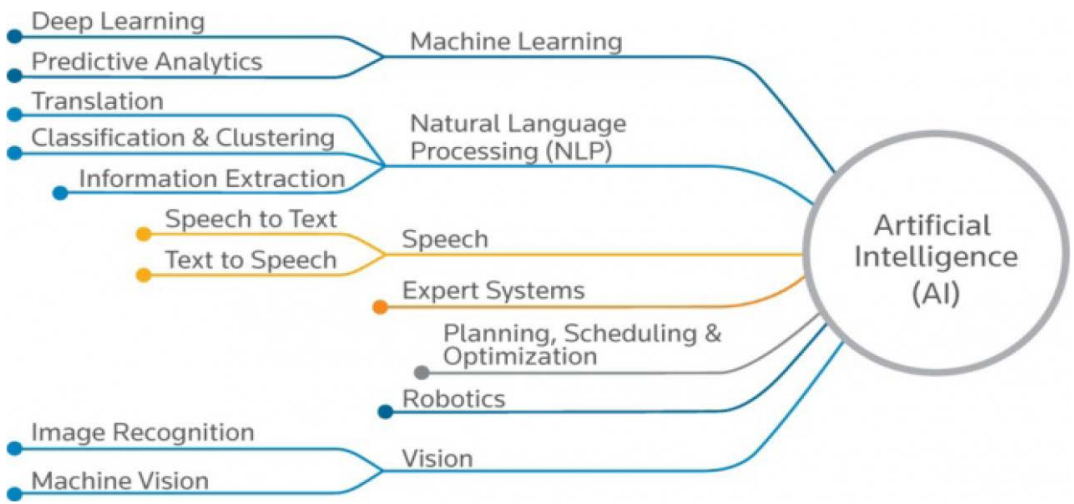

Source: Neota Logic.

(blogs.thomsonreuters.com) 


\section{Risks and Fears}

Mostly, we don't need to enumerate the advantages of Artificial Intelligence and its usefulness for our modern life today. This paper in deed, in its entirety is talking about its importance, advantages and benefits which cannot be dispensed from now on. However, it's maybe useful to point out some fears or damages we may encounter in present or in future due to AI, whether it is at the level of individuals or societies.

a - The intrinsic fear of the matter of AI is that human can give these machines a lot of intelligence via codes and programs, but he cannot give them any kind of virtue, feelings or ethics. As it's known that intelligence isolated from virtue could be very dangerous. Thus, such kind of worries are really justified.

b - Costs incurred in production, maintenance and repair of AIbased machines are very high, especially when it's necessary to update them continuously to meet the renewable demands. (techspirited.com)

c - Unemployment is the axiom direct result of an irrational process of replacement of human workers with smart machines. In the light of the fact that one smart machine is capable of working efficiently instead of dozens of workers with lower costs. man can imagine how huge this disaster is that millions of workers all over the world are about to loose their living source with their families, especially with such political and economical systems. It is obvious that unwise employment of smart machines in labor sector will lead to a problem needs decades before being solved.

$\mathrm{d}$ - The more humans depend on smart machines, the lesser lateral thinking and multitasking ability they are going to have. With the heavy application of AI, humans will become overly dependent on machines, losing their mental capacities. (techspirited.com)

e - If the control of smart machines goes in the wrong hands, it may cause evil and destruction, because machines won't think before acting as soon as they receive the order. i.e., when the programmer of such machines is a bad one, we may expect a really mass destruction. (wisestep.com) 
Many other risks and fears could be discussed in this course. However, the points have been mentioned above give a collective view of risks and fears representing the main domains that humanity worries are involved in.

\section{Artificial Intelligence Governance}

As we can obviously notice, most of our life's sectors are rapidly adopting technologies of AI, autonomous systems and algorithmic decision-making, which have been intervening lives of billions many years ago (E. Horvitz; 2017).

Google and Facebook have been using AI-based filtering algorithms to control access to information, as well as self-driving cars. Various applications including security and safety decision making systems rely heavily on AI- based face recognition algorithms. All of these aspects have been mentioned are just simple examples of how AI-based applications are controlling our lives now (S. Wachter, 2017).

Based on the above, governments in the first place, supported by civil society, private sector and academia must save no effort in such workshops in which the discussion, assessment and research are carried out to develop a set of mechanisms, specifications and regulations that must be sponsored by governments for the sake of minimizing the risks and all downsides of AI. i.e., to keep AI and autonomous systems under human control; if we are really interested in adopting values of transparency and accountability of AI-based systems.

Governance of AI will not only protect humanity from risks and domination of AI-based systems, but it is the right way to find out and commit the best paths to maximize the desired benefits of this grace that humanity hasn't been granted before. In one word or another, governance of $\mathrm{AI}$ is the way to maximizing and maintaining this grace and preventing it from becoming a destructive curse.

A roadmap made by experts has identified some issues must be taken in consideration in our way to AI governance, where answering the questions posed by these substantive critical issues will outline the most important features of the road we must take to reach our goal of the ideal governance, some of these issues are: 
a - Justice and Equality: Is it really possible to design AI system to reflect human values?

b - Use of Force: After involving AI- based systems in making decisions about the use of force, autonomous weapons for example. Is the human control necessary? To what extant if it is? and the most sensitive question: Who will be responsible for the AIbased outputs?

c - Safety and certification: How do we define and validate safety thresholds?

d - Privacy: What are the privacy implications and new privacy threats of next generation technologies, in terms of government surveillance and corporate influence over customers for example?

e - Displacement of Labor and Taxation: To what extant smart machines will replace jobs of humans? And: What are the effects of AI on public if workers lost their living source and robots don't pay taxes?

(R. Calo; 2017)

\section{Artificial Intelligence Governance in KSA}

"I am very honored and proud of this unique distinction". with this speech, Sophia the robot spoke to the audience at The Future Investment Initiative in Riyadh after she was granted the Saudi citizenship, on Wednesday the $25^{\text {th }}$ October 2017. (Business Insider)

As Sophia - the social humanoid robot developed by Hong Kong based company Hanson Robotics and was activated on February 2016 (Mallonee, Laura, 2018) - has become the first robot to be granted a citizenship in the history, The Kingdom of Saudi Arabia is the first country in the history to grant a citizenship to a robot. (Business Insider)

This historic event shows to what extent the Kingdom of Saudi Arabia looks at AI as an important strategic choice, it outlines the next era in which the Kingdom intends to hold a high distinguished position among the world's nations that consider AI as a great hope and the new foundation for the new technological revolution that promises humanity better solutions in all fields. 
In line with this creative spirit, Kingdom of Saudi Arabia has decided to adopt Digitization and AI as the key enablers of its new era, as it explained in its Vision 2030, working on its project of building an Information and Communications Technology (ICT) infrastructure for the $21^{\text {st }}$ century (WIPO MAGAZINE).

Ministry of Technology, Industry and Digital Capabilities is working now to enhance the digital capabilities of workforce to align with AI applications such as the Internet of Things (IOT) and Blockchain, which is leading the kingdom to adopt a major educational reform, that is why the Ministry of Technology is working side by side with Ministry of Education to meet changing demands of the workplace, through introducing digital skills in K12 education, along with reforming university curricula to make it matching future needs.

At present, a wide number of governmental and private agencies are working together to support the idea of entrepreneurship and innovation. One of this efforts' reflection is the building of a network of innovation labs where students and entrepreneurs can explore ideas, learn about and test innovative business models.

The Ministry of Technology has recently launched an innovation platform called FekraTech, encouraging every concerned one to share his innovative ideas; in one round, about 4000 proposals have been received, many of these proposals where really valuable, for example, a proposal of invention called Nahla, an AI-based chatbot helps people of chronic diseases to learn about and better manage their condition. (WIPO MAGAZINE)

Kingdom of Saudi Arabia has been calling for global contractors in technology field few years ago, Amazon, IBM and Alibaba are negotiating now for partnerships with kingdoms officials, while the Chinese tech conglomerate Huawei is already committed to training of 1500 local engineers. Kingdom aims to approach a better life for Saudi people through all of these steps.

However, efforts have been made for the sake of the employment of AI in the improvement of life conditions of Saudi people is not limited to government agencies, but private sector and community institutions particularly universities have their important role. 
An Important example of the of this approach is the matter of managing crowd flows near Kaaba during pilgrim, in the aftermath of 2015 tragedy in which more than 2000 pilgrims lost their lives as a result of rush in Mina, two professors of Umm Al-Qura University introduced a research project using computer vision to manage crowd flow near Kaaba. "Deep learning algorithms can count the number of people in a scene with up to 97.2 percent accuracy. A heat map signals a warning when density exceeds $4-5$ people per square meter, and the system can also monitor crowd circulation speed for safety purposes" (Medium.com)

Kingdom of Saudi Arabia has sensed recently the growing fatal danger of traffic accidents on kingdom's roads. Therefore, and in line with its approach to tackle society's troubles throughout creative intelligent solutions, KSA government has begun a long-term cooperation process with technology companies whether they are local or global to get the best solutions, one of these companies is Hazen company.

Hazen company has received a government contract to work on urban safety, Hazen is using computer vision to enforce seat belt wearing and spot traffic violations. Moreover, it is working on building advanced traffic cameras with the capability to detect dangerous driving behavior through video analysis. (Medium.com)

It is worth mentioning that Hazen is only one of many companies operating in the Saudi market, for more specification, it is one of the companies incubated by Wadi Makkah Company investing in tangible and intellectual assets and in knowledge-based production, which is totally owned by Umm Al Qura University (UQU). Another example of such companies is Traffixity Company, that "provides and intelligent transportation system implementable in cities using advanced computer vision and offers advanced sensors to support traffic decision making. (WadiMakkah.com)

\subsection{Post Oil Era}

It is not a secret that countries of Gulf Cooperation Council (GCC) have always been dependent on oil revenues. Not long ago, 
decision-makers in these countries noticed that oil exports would not be that strategy that would guarantee their countries lasting growth and prosperity. This fact was further demonstrated by the recent oil price crash due to the stormy political crisis in the region. Consequently, many are looking to AI as the "oil" of the new phase; in other words, the basis on which these countries will depend on in the post-oil era.

Crown Prince Mohammed Bin Salman has pointed clearly to this fact in World Economic Forum, 2016 when said "We have developed a case of oil addiction". (World Economic Forum)

A study conducted by a group of researchers, found that Artificial Intelligence's impact on Saudi Arabia economy is significant. AI can add up to 1.1 percentage points to Saudi Arabia's economic growth rate, where the potential Gross Value Added (GVA) of that AI augmented growth is about 215 Billion Dollars.

\section{Al as a new factor of production can lead to significant growth opportunities for these economies}

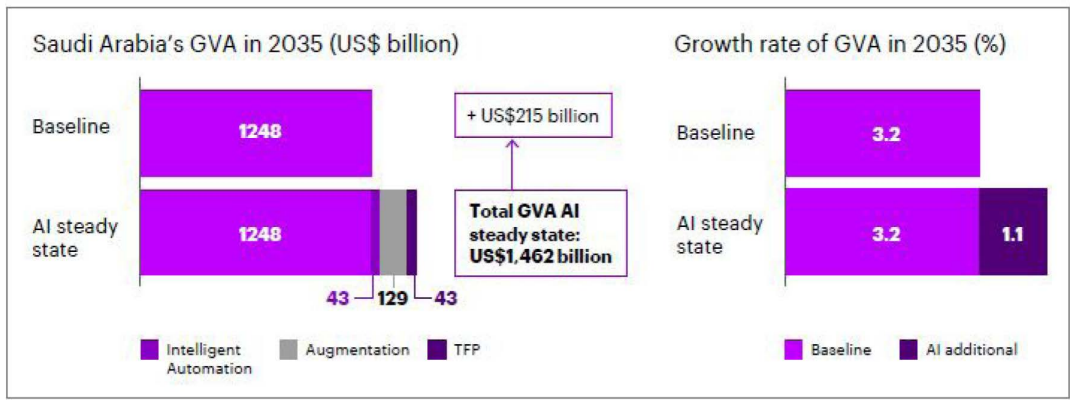

As the researchers tended to determine the potential sector- specific impact of artificial intelligence on the economies of the Middle East, they looked at 13 industries in Kingdom of Saudi Arabia, combining the results with their AI Modeling with industry size data. The model showed that "in absolute dollar terms, Manufacturing, Public Services and Professional Services will benefit the most in Saudi Arabia, with boosts in industry GVA of $\$ 37$ billion, $\$ 67$ billion and $\$ 26$ billion respectively by the 2035 year. 


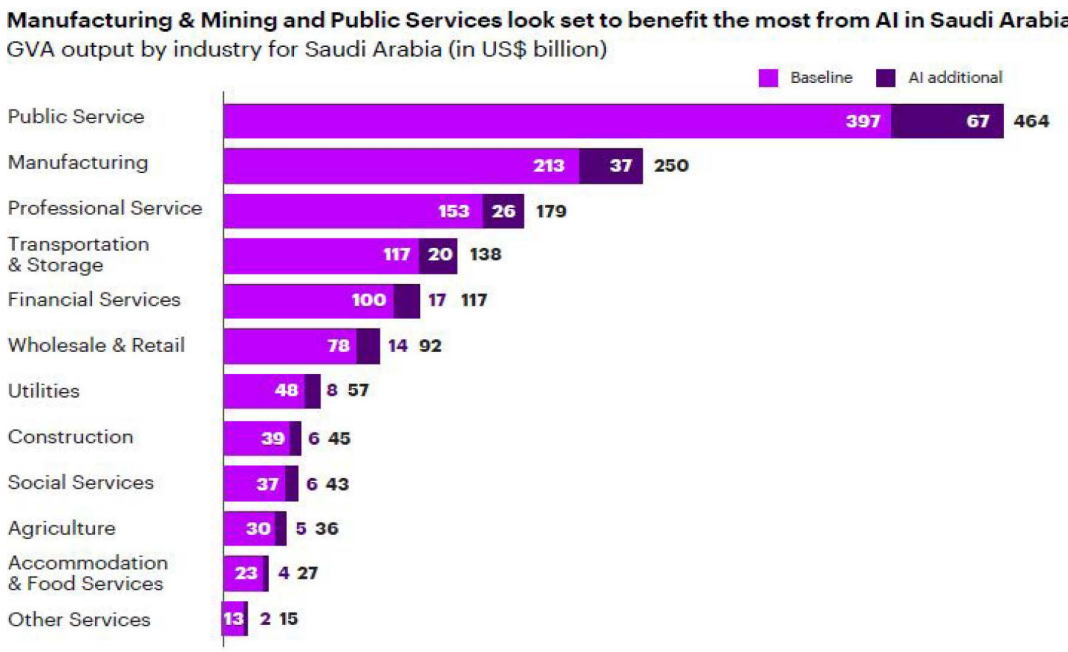

Source: Accenture and Frontier Economics

(Amr Elsaadani, Mark Purdy, Elizabeth Hakutangwi.; 2018)

\section{NEOM Project}

"The land of the future, where the greatest minds and best talents are empowered to embody pioneering ideas and exceed boundaries in a world inspired by imagination" (NEOM Official Site), with these words, NEOM's founding council began its presentation of this giant project's vision. It is needless to say that a "world inspired by imagination" needs much more than human's traditional methods, i.e., Artificial Intelligence is a basic player indeed.

"The acronym NEOM is composed of two parts: The first is derived from the Latin word neo (new), and, the second, letter m, represents the Arabic word for future: mostaqbal. Collectively, NEOM spells "New Future!"”. (INTERNATIONAL POLICY DIGEST)

NEOM is a Saudi project for a smart and tourist cross-border city, located in the fart north-west of Saudi Arabia in Tabuk, including a marine land located within the Egyptian and Jordanian borders of a total area of 26,500 km2 extending $460 \mathrm{~km}$ on the coast of Read Sea. The construction of NEOM is expected to begin in 2020. 
This project was announced by Crown Prince Mohammed bin Salman to meet his ambitious aspirations to see the Kingdom of Saudi Arabia as a leading global model in tourism and intelligence for the year 2030, with over 500 billion dollars in funding in the coming years seeking the ways of cooperation and investment with a wide network of international investors and innovators. (Bloomberg)

Experts describe the end goal of this giant project as "to shift from oil to high tech and put Saudi Kingdom at the forefront to technological advances. This is the post-oil era" (Inverse).

Perhaps, this description points to the importance and depth of this project, which forms a long-term strategic challenge of experts and decision makers in the kingdom, to move the kingdom from one age to another, and from a place among the others to another one at the forefront of the globe. All of this gives an important impression on the interest the kingdom attaches to this project.

\subsection{View}

"We want the main robot and the first robot in Neom to be Neom itself. Robot number one. Everything will have a link to artificial intelligence, to the Internet of Things, ..., So nobody can live in Neom without the Neom application we'll have, or visit Neom" (Saudi crown prince to Bloomberg).

As the chairman of the founding council, Crown Prince Mohammed Bin Salman described his collective view of NEOM, it is simply that the artificial Intelligence will be the Backbone of NEOM City.

\subsection{NEOM SECTORS}

According to the official website of NEOM, NEOM is adopting eleven sectors in which it will make a big difference, to be as never seen before:

1 - Energy and Water

2 - Mobility

3 - Biotech

4 - Food

5 - Advanced Manufacturing 
6 - Media

7 - Entertainment

8 - Technological and Digital Science

9 - Tourism

10 - Sport

11 - Living as NEOM's Foundation

According to NEOM's officials, each one of the aspects - mentioned above - will be closely linked to AI, via different applications that are almost in tune with human desires and requirement. This is the philosophy of NEOM.

In order to highlight the principle of using artificial intelligence, a brief discussion is better to be carried bout for some of these aspects:

\subsubsection{Mobility}

"Across land, air and sea, NEOM will become a global hub of connectivity". These words described NEOM's vision of mobility, it is how to create an industrial city moving with the least human engagement. To convey containers from factories to ports automatically, to secure an easy automated bath to warehouses approaching the least costs. This is true also for different means of transportation in NEOM. (Bloomberg).

\subsubsection{Advanced Manufacturing}

In the light of the fact that investments in Robotics sector are about 3.6 Billion dollars with grow rate of $16 \%$ until 2030, NEOM intends not only to innovate in nanotechnology, 3D printing, sensors. etc., but to start from scratch to create innovative systems supporting innovations of research and development.

Fore example, NEOM experts suggest that smart traffic technology could reduce traffic in NEOM by $25 \%$ and congestion by $40 \%$, such a simple example shows to what extent NEOM staff relies on AI and its applications do make things different.

\subsubsection{Technological and Digital Science}

In this area, NEOM openly declares itself as a nucleus of the new era of AI, and a global platform from where all concerned scientists all 
over the world will launch their researches, analytics and innovations since it is opened for all of them.

This will be accomplished by NEOM's policy of adopting the "Open-Source Platform". Consequently, NEOM grants top concerned scientists all over the world their first and unique opportunity to use NEOM itself as a test ground for their activities and innovations.

\section{Living as NEOM's Foundation (Livability)}

The basis of planning NEOM by the founding council was conceiving it as the "Utopia" of the modern life. This ambition is exemplified by offering an ideal lifestyle in all aspects, through adopting a modern architecture capable of providing high levels of luxury for its residents.

This luxury is translated into all spaces it is going to contain, entertainment, sport, lush green and open spaces, quality of life, safety and technology, all of this besides excellent economic opportunities.

As the chairman of the founding council, Prince Mohammed Bin Salman was trying to explain his view about NEOM, he stated that his goal is to achieve in reality what is still only in minds, i.e., ideas out of the box. For example, no one of NEOM needs to go to supermarkets to buy things, for technological solutions will enable NEOMs to have their stuff in houses effortlessly. (Saudi crown prince to Bloomberg).

The main notion/feature of NEOM is that everything is simply Intelligent and connected, for example, a NEOM's resident will have his medical file linked with his home supply, car, family and everything else, where this continuous connection will lead to a self-developing system that provides the resident with the best for his interest. (Bloomberg)

\section{References}

1. See "A Brief History of Computing" at AlanTuring.net

2. Smith, C., McGuire, B., Huang, T., \& Yang, G. (2006, December). History of Artificial Intelligent [Scholarly project]. Retrieved November 20, 2017.

3. Norvig \& Russell $(2003$, p. 948$)$ claim that Turing answered all the major objections to AI that have been offered in the years since the paper appeared. 
4. Crevier (1993, pp. 49) writes "the conference is generally recognized as the official birthdate of the new science.

5. McCorduck, Pamela (2004), Machines Who Think (2nd ed.), Natick, MA: A. K. Peters, Ltd.

6. Crevier 1993, pp. 52-107, Moravec 1988, p. 9 and Russell \& Norvig 2003.

7. Crevier, Daniel (1993), AI: The Tumultuous Search for Artificial Intelligence, New York, NY: BasicBooks.

8. Stone, P., Brooks, R., Brynjolfsson, E., Calo, R., Etzioni, O., Hager, G., Hirschberg, J., Kalyanakrishnan, S., Kamar, E., Kraus, S. and Leyton-Brown, K., 2016. Artificial intelligence and life in 2030. One Hundred Year Study on Artificial Intelligence: Report of the 2015-2016 Study Panel.

9. Liao Lin, Patterson Donald J, Fox Dieter, Kautz Henry (2007). Learning and inferring transportation routines. Intelligence, Volume, April 2007, Pages 311-331.

10. Automated Vehicles: Policy Implications Scoping Study by Jason Wagner Trey Baker Ginger Goodin and John Maddox Report 60045100029-1 Project Title: Policy Implications of Automated Vehicles on Texas Highways Sponsored by Southwest Region University Transportation Center Texas A\&M Transportation Institute The Texas A\&M University System College Station, Texas 77843-3135 January 2014

11. Nilsson Nils J. (2010). The Quest for Artificial Intelligence. Cambridge University Press http://www.cambridge.org/us/

12. LeighAnne Olsen, Dara Aisner, and J. Michael McGinnis, eds., 2007), accessed August 1, 2016 "Institute of Medicine (US) Roundtable on Evidence-Based Medicine," The Learning Healthcare System: Workshop Summary. (Washington (DC): National Academies Press (US)

13. https://www.techemergence.com/artificial-intelligence-and-security-applications/

14. https://blogs.thomsonreuters.com/answerson/artificial-intelligence-legal-practice/

15.https://techspirited.com/pros-cons-of-artificial-intelligence

16. https://content.wisestep.com/advantages-disadvantages-artificial-intelligence/

17. E. Horvitz, “AI, People, and Society,” Science, vol 357, no. 6346, 2017, p. 7. 
18. S. Wachter, B. Mittelstadt, and L. Floridi, "Transparent, Explainable, and Accountable AI for Robotics," Science Robotics, vol. 2, no. 6, 2017; doi:10.1126/scirobotics.aan6080.

19. R. Calo, "Artificial Intelligence Policy: A Roadmap," Social Science Research Network (SSRN), 8 Aug. 2017.

20.https://www.businessinsider.com/sophia-robot-citizenship-in-saudiarabia-the-first-of-its-kind-2017-10

21.http://www.wipo.int/wipo_magazine/en/2018/05/article_0002.html

22.https://medium.com/syncedreview/ai-as-the-new-oil-saudi-arabias500-billion-smart-city-f7b63f7c9423

23. http://wadimakkah.sa/en/established-company/\#1520497637770-5c9e6ab5-adc6

24. World Economic Forum, "Which economies are most reliant on oil?", May 10, 2016

25. Amr Elsaadani, Mark Purdy, Elizabeth Hakutangwi.; Pivoting With AI. 2018. www.accenture.com/FinancialServices; https://www.accenture. com/t20180509T033303Z_w_/us-en/_acnmedia/PDF-77/Accenture-Impact-AI-GDP-Middle-East.pdf

26. Mallonee, Laura (2018-03-29). "Photographing a Robot Isn't Just Point and Shoot]". Wired. Retrieved 2018-10-10.

27.http://www.neom.com/

28.https://intpolicydigest.org/2018/03/18/neom-and-project-code-are-unstoppable/

29. https://www.bloomberg.com/news/articles/2017-10-24/saudi-arabiato-build-new-mega-city-on-country-s-north-coast

30. Saudi Arabia Reveals NEOM, its Planned Futuristic Megacity". Inverse. October 25, 2017.

31.https://www.bloomberg.com/news/articles/2017-10-26/saudi-arabiacrown-prince-details-plans-for-new-city-transcript

\section{DATA ABOUT THE AUTHOR}

\section{Rana Ashehri}

King Abdulaziz University

Al Ehtifalat St, Jeddah 21589, Saudi Arabia

raabdul1122@gmail.com

ORCID: 0000-0002-6507-2844 